\title{
Editorial
}

\section{In the December 2013 issue}

I n this issue of Dementia $\mathcal{E}$ Neuropsychologia, we are publishing one review, ten original papers, one case report and the first paper inaugurating the History Note section of the journal, which shall be edited by Prof. Eliasz Engelhardt.

Brucki reviewed the epidemiology of mild cognitive impairment (MCI) in Brazil concluding that Brazilian data remain scarce hampering comparisons with other countries. Her findings should prompt investigators to undertake and publish investigations on the epidemiology of MCI in Brazil.

Batista et al. investigated the test-retest reliability of tests of the Brazilian version of the CERAD battery. Better retest scores were observed with a 20day interval, suggesting that retest should be applied after longer intervals or by using alternate forms of the tests.

Foss et al. evaluated the performance of middleaged and elderly Brazilian on the Mattis Dementia Rating Scale (DRS) to propose norms for improving the diagnostic accuracy of this scale. Age and educational level were taken into account as these two factors have strong correlation with DRS scores.

Silagi et al. investigated the effects of social exposure on communication in the elderly. The authors found that sociodemographic features were associated with communication habits (partners and communication time) and that time devoted to communication with friends may indicate cognitive difficulties

Lima-Silva et al. carried out the translation and cross-cultural adaptation of the Frontotemporal Dementia Rating Scale (FTD-FRS) for use in Brazil. This scale was considered suitable to aid in staging and determining disease progression for BrazilianPortuguese-speaking patients with frontotemporal dementia.

Souza-do-Carmo et al. investigated renal function in elderly with and without Alzheimer's disease (AD). Their conclusions were that patients with $\mathrm{AD}$ have lower glomerular filtration and a greater incidence of microalbuminuria, not associated with higher vascular risk factors. These are preliminary findings and further studies should be conducted to investigate the renal function of elderly with $\mathrm{AD}$.

Castro-Costa et al. investigated the association between nutritional status and cognitive impairment in community-dwelling elderly. The authors found that cognitive impairment was associated with fat mass measures in the group aged between 60 and 69 yet with muscle mass measures in the group aged over 70 .

Rezende et al. compared the Cognitive Abilities Screening Instrument-Short Form (CASI-S) with Mini-Mental State Examination (MMSE), and the Functional Activities Questionnaire for the diagnosis of dementia in illiterate elderly. The authors found a high correlation between the CASI-S and MMSE and suggested further studies to evaluate the use of CASI$S$ for dementia diagnosis in populations with a heterogeneous educational background.

Marroni et al. evaluated the normal values of cerebral perfusion using single-photon emission tomography (SPECT) with ${ }^{99 m}$ Tc-ethyl cysteinate dimmer. This method may allow further quantification of SPECT data, specifically the comparison of patients with normal controls.

Hur \& Caixeta evaluated more complex language tasks in two cases of semantic dementia, finding that the interpretations of proverbs and metaphors as well as comprehension of ironic statements were disturbed. The possible causes of these symptoms are discussed.

Fortes et al. reported two cases of neurosarcoidosis presenting as rapidly progressive dementia. The diagnostic criteria and differential diagnosis of this condition are discussed.

In this inaugural paper of the History Note section, Engelhardt reviewed the pioneering stud- 
ies on basal formations of the brain, particularly of Meynert's basal nucleus, emphasizing the works of Meynert and also of Reil, Gratiolet and Koelliker.

Champs et al. reported a case of a patient with progressive paraparesis and cognitive changes referred with the possible diagnosis of multiple sclerosis, whose serum and cerebrospinal fluid were positive for HTLV-I infection. The authors discuss the

\section{HISTORY NOTE: A NEW SECTION}

Tistorical Note is a new permanent section chologia.

One might ask: why study history of neurology and related issues, when history is largely regarded as events of the past that are outdated and no longer valued in practical terms?

The answer is clear: our present is based on our past, and we should be fully aware of where we have been, and where we are, before we venture into the future. Traditional sayings such as "If I have seen further it is by standing on the shoulders of giants", among others, clearly denote this view.

Studying history develops a critical view of present achievements. History allows us to understand the pioneering researchers, their lives and struggles, their reasoning, their successes and failures, the relentless pursuit of their aims in an unabated fashion, their achievements, how they paved the way in a poorly charted brain, their egos...

To view past achievements as meritless is folly, as oftentimes, ill-described structures can become, sometimes decades or centuries later, advance techniques and flourishing functional systems.

History allows us to explore the roots of today's ideas and practices and thus to appreciate both the ephemeral nature of knowledge and the need for continual learning. A sense of humility and of constructive criticism for personal endeavors develops in those who look to past achievements.

Some authors that have inspired these considerations should be cited: such as Cordell, 1904; Guthrie, differential diagnosis between these two conditions emphasizing the dissimilar patterns of cognitive impairment.

\section{Ricardo Nitrini}

Editor-in-Chief
1945; Thornton, 1987; Finger, 2000; Koehler et al., 2000; Maienschein,2000; Gooday et al., 2008; Roche et al., 2009; Gomes and Teive, 2012.

The instructions for submitting manuscripts are very flexible: the main text may have up to 1,500 words, with an abstract, including keywords, containing about 150 words, references may number up to 30 , and, optionally, up to 2 figures and 2 tables or boxes may be included. General additional information can be obtained from the site of the journal.

One should keep in mind that writing history requires the reading of original texts (primary) which represents a firsthand re-reading and re-interpretation of the writings, as it is known that very often the filter of time and of secondary works may distort or even miss out parts of the original findings, resulting in errors which may frequently be accepted as truths, and perpetuated for long periods.

Call for papers: After these reflections and instructions, the editor hereby invites all professionals from our neurological and cognitive community to submit a paper on history. Clearly, in view of the characteristics of this journal, the focus should center mainly on authors and their works that have influenced thinking on cognition, behavior and related neuroscientific issues.

Now is the time to begin.

\section{Eliasz Engelhardt}

Editor, History Note section 